\title{
Japan's Lost Decade: Does Money have a Role?
}

\author{
Fabio Canova \\ ICREA-UPF, AMeN and CEPR University of Bern *
}

Final draft: November 2009

\begin{abstract}
We study the contribution of the stock of money to the macroeconomic outcomes of the 1990s in Japan using a small scale structural model. Likelihood-based estimates of the parameters are provided and time stabilities of the structural relationships analyzed. Real balances are statistically important for output and inflation fluctuations and their role has changed over time. Models which give money no role give a distorted representation of the sources of cyclical fluctuations. The severe stagnation and the long deflation are driven by different causes.
\end{abstract}

JEL classification numbers: E31, E32, E52,

Key words: Money, Japan's Lost decade, structural model, deflation.

${ }^{*}$ We thank an anonymous referee, the participant of the TRIO workshop and Andrew Levine for comments and suggestions. Discussions with Toni Braun and Etsuro Shioji are gratefully acknowledged. The financial support of the Spanish Ministry of Education through the grant SEJ-2004-21682-E and of the Barcelona Graduate School of Economics are gratefully acknowledged. 


\section{Introduction}

The poor macroeconomic performance of Japan in the 1990s has been the subject of an extensive investigation in the literature. Two reasons contribute to make this episode unique from the point of view of post-WWII history: the extremely long and severe output stagnation, comparable only to the one experienced during the 1930s, and the persistent deflation, unprecedented in developed countries. The extraordinary length of the recession makes the experience an excellent laboratory to study movements in economic activity in the medium run, their sources and the mechanisms able to stretch over time temporary exogenous disturbances. The prolonged deflation and the negligible interest rates that accompanied it, on the other hand, brought back on the front stage of academic research dormant liquidity trap theories (see e.g. Krugman, 1998) and shifted the focus of policymaking activities from monetary to fiscal and other types of stabilization policies.

The literature has provided several explanations for the so-called "Lost decade". They range from the existence of important monetary and fiscal policy mistakes (see Ito and Mishkin, 2004), to depressed investment activities following the asset bubble of the late 1980s; to a slowdown in total factor productivity coupled with changes in workweek regulations (see Hayashi and Prescott, 2002 and Esteban Pretel et al., 2008); to misallocation of resources toward unproductive firms (see Caballero et al., 2008). In general, most analyses emphasize problems with the real or the financial intermediation side of the economy and the extremely slow response of policymakers to economic conditions. Even studies which are monetary in nature (such as Braun and Waki, 2006, or Eggertsson and Woodford, 2003) give the stock of money no role in, e.g., amplifying fluctuations generated by unexpected disturbances. There is an important reason for this state of affairs. The majority of the monetary models nowadays employed for business cycle analysis and for optimal policy design share the feature that the stock of money has a marginal importance in determining the equilibrium of the economy. Often models make no reference whatsoever to monetary aggregates, and when they do, they use a specification where a money demand function determines how much money needs to be supplied, given predetermined levels of output, inflation, the nominal rate,

etc. Hence, the quantity of money plays no role in amplifying and/or stretching over time output and inflation fluctuations.

Why should money be meaningfully included in the theoretical framework used to 
study medium term fluctuations and to discuss the state of the Japanese economy in the 1990s? We can think of, at least, three reasons for why omission of money may induce distortions in the interpretation of the evidence. First, while it is relatively well known that money has little or no correlation with real variables and inflation at business cycle frequencies, this correlation is considerably larger when longer cycles are considered. The correlation between per-capita cyclical M2 and per-capita cyclical output in Japan since 1980 is only 0.10, when cycles of 6 to 24 quarters are considered, but increases to 0.90 , when cycles of 24 to 44 quarters are considered. Second, to explain a decade long stagnation one cannot rely on a decade long sequence of negative shocks. Instead, some mechanism amplifying temporary disturbances and transforming them into medium term fluctuations is needed. The literature has provided suggestions along these lines (see e.g. the R\&D motive of Comin, 2008), but the ability of these mechanisms to explain the experience appears to be limited. The stock of money could potentially play such a role if, for example, there are complementarities in production or in the utility function. Third, the emphasis that the Bank of Japan has given to monetary aggregates, as instruments to escape the prolonged deflation in the last few years, and the even more recent Federal Reserve Bank and European Central Bank policies of quantitative easing, suggest that, at least in some policymakers' mind, the stock of money may matter for both output and inflation fluctuations.

This paper examines the role of the stock of money in amplifying fluctuations in output and inflation in Japan over the last 30 years; measures whether and in what way money has contributed to transform the slowdown of the beginning the 1990s into the "Lost decade" and studies which structural disturbance contributes to explain the path of output and inflation in the 1990s. The analysis is conducted using a variant of a small-scale structural New-Keynesian model. We first show what money can do in such a model, simulating conditional paths for output and inflation in specifications where money has a role and where it does not. We then estimate the structural parameters of the model using maximum likelihood (ML) techniques, examine the stability of the structural relationships and construct historical decompositions to analyze sources of fluctuations in output and inflation. Since the small structural model we employ is incapable of accounting for all aspects of such a complicated experience, we regard our work as contributing to the development of a better understanding of the reasons behind protracted periods of slowdown in economic activity rather than testing one or more causes of the "Lost decade". Nevertheless, our structural analysis allow us to 
comment on certain explanations put forward in the literature.

For the sake of presentation, we organize the discussion of our findings around four broad questions. First, does money have a role in explaining fluctuations in output and inflation in Japan since 1980? Second, is there a change in the importance of money over interesting subsamples? Third, does the model account for the "Lost decade" via changes in the structural parameters or via changes in the distribution of the shocks? Fourth, what disturbances could be jointly responsible for the output stagnation and the deflation observed in the 1990s? Our results are as follows.

First, money is statistically and economically important in characterizing cyclical fluctuations in output and inflation in Japan. Money matters both directly, through its effects on the Euler equation and the Phillips curve, and indirectly, through the Central Bank determination of the nominal interest rate. Money plays a role because it alters both the marginal rate of substitution between consumption and leisure and the intertemporal rate of substitution of consumption at different points in time and because, when reacting to nominal balances, the monetary authority indirectly reacts to inflation. These channels help to amplify the magnitude of cyclical fluctuations that the model can account for and make their persistence stronger. The evidence we collect suggests that neglecting money may create important statistical biases and relevant inferential mistakes when interpreting the 1990s in Japan.

Second, the role of money has changed over time and our estimates indicate that it may have mattered most in the 1990s. Interestingly, it played little role over the last ten years. While this outcome may be taken as a sign of misspecification - in the 1990s money may be proxying for a mechanism which is missing from the model - we have found no evidence that money stands in for credit, asset prices or standard omitted suspects. Thus, the slow expansion of the quantity of money in circulation may have contributed to transform the downturn of the beginning of the 1990s into a severe output stagnation and a moderate inflation into a deflation.

Third, our model accounts for Japan's "Lost decade" through changes in the economic parameters regulating the private sector behavior and the monetary policy rule and in the auxiliary parameters regulating the persistence and the volatility of the exogenous disturbances. This is consistent with what the literature on the Great Moderation in the US has found, using similar models (see e.g. Canova, 2009). However, changes in the distribution of the shocks appear to be a much more important mechanism to account for the experience than changes in the economic parameters. 
Fourth, the prolonged fall in output and the persistent deflation are due to different causes. Shocks that affect the intertemporal marginal rate of substitution in the economy are crucial to explain the path of output in the 1990s. Shocks that alter the marginal cost of production, on the other hand, primarily drive inflation movements during the decade. Interestingly, technology shocks have little to do with the output stagnation of the 1990s. Since it is well known that Solow residuals are poor proxies for technological variations, the idea that TFP changes are responsible for the "Lost decade" should be probably reconsidered. Finally, monetary policy is in part to blame for the poor macroeconomic outcomes. Monetary policy has been very restrictive, at least up to 2003, and this has contributed to keep both the recession and the deflation going for a number of additional years. The situation appears to have changed since 2003 but only marginally.

The rest of the paper is organized as follows. The next section presents the theoretical model used to organize the data and shows what money can do to output and inflation fluctuations. Section 3 describes the data and its sources. Section 4 presents the results for the full sample and for selected subsamples, and examines how the model deals with the "Lost decade". Section 5 studies what drives the path of output and inflation in the 1990s. Section 6 concludes.

\section{The theoretical framework}

Our model builds on the basic New-Keynesian model without capital accumulation described in Gali (2008). We extend this simple structure in three ways. First, we allow for habit in consumption. Second, we allow but do not require the stock of money to play a role in the determination of output and inflation, by making real balances enter non-additively in the utility of the representative agent. Third, we permit the growth rate of nominal balances to enter the Central Bank rule. Apart from giving money an indirect role in the model, the specification we choose allows us to deal with the problem of appropriately specifying a policy rule when the nominal rate hits the zero bound (see section 4.5 for a discussion). We have also considered the possibility that the growth rate of the exchange rate also enter the policy rule. In this case, we treated Japan as a small open economy and the exchange rate was determined, in equilibrium, by an interest parity condition. We present results only for the closed economy version of the model because the exchange rate seems to play no role in the analysis and the 
international (financial) feedbacks seems relatively small.

\subsection{The model}

Since the economy is quite standard, we only briefly describe its features. There is a representative household, a representative final good producing firm, a continuum of intermediate goods firms each producing a differentiated good $i \in[0,1]$ and a monetary authority. At each $t$ the representative household maximizes

$$
E_{t} \sum_{t} \beta^{t} a_{t}\left[U\left(x_{t}, \frac{M_{t}}{p_{t} e_{t}}\right)-\eta n_{t}\right]
$$

where $x_{t}=c_{t}-h c_{t-1}, 0<\beta<1, h, \eta>0$ subject to the sequence of constraints

$$
M_{t-1}+T_{t}+B_{t-1}+W_{t} n_{t}+D_{t}=p_{t} c_{t}+\frac{B_{t}}{R_{t}}+M_{t}
$$

where $c_{t}$ is consumption, $n_{t}$ are hours worked, $p_{t}$ is the price level, $M_{t}$ are nominal balances, $W_{t}$ is the nominal wage, $B_{t}$ are one period nominal bonds with gross nominal interest rate $R_{t}, T_{t}$ are lump sum nominal transfers at the beginning of each $t$ and $D_{t}$ are dividends distributed by the intermediate firms. $a_{t}$ and $e_{t}$ are disturbances to preferences and to the money demand, whose properties will be described below. Let $m_{t} \equiv \frac{M_{t}}{p_{t}}$ denote real balances and $\pi_{t} \equiv \frac{p_{t}}{p_{t-1}}$ the gross inflation rate during period $t$.

The representative final good producing firm uses $y_{t}^{i}$ units of intermediate good $i$, purchased at the price $p_{t}^{i}$ to manufacture $y_{t}$ units of the final good according to the constant returns to scale technology $y_{t}=\left[\int_{0}^{1}\left(y_{t}^{i}\right)^{(\theta-1) / \theta} d i\right]^{\theta /(1-\theta)}$ with $\theta>1$. Profit maximization by these firms yields demand functions of the form

$$
y_{t}^{i}=\left(\frac{p_{t}^{i}}{p_{t}}\right)^{-\theta} y_{t}
$$

so that $\theta$ measures the constant price elasticity of demand for each intermediate good. Competition within the sector implies that $p_{t}=\left(\int_{0}^{1}\left(p_{t}^{i}\right)^{1-\theta} d i\right)^{1 /(1-\theta)}$.

An intermediate goods producing firm $i$ hires $n_{t}^{i}$ units of labor to produce $y_{t}^{i}$ units of intermediate good using the production function $y_{t}^{i}=z_{t} n_{t}^{i}$, where $z_{t}$ is an aggregate productivity shock. Since intermediate goods substitute imperfectly for one another in producing finished goods, the intermediate firms act as monopolistic competitors in their pricing decisions. We assume that, when firms change prices, they face the following cost of adjustment, measured in terms of finished goods:

$$
\frac{\phi}{2}\left(\frac{p_{t}^{i}}{\pi^{s} p_{t-1}^{i}}-1\right)^{2} y_{t}
$$


where $\phi>0$ and $\pi^{s}$ measures steady state inflation. The problem faced by the representative firm in setting its price is therefore to maximize

$$
E_{t} \sum_{t} \beta^{t} a_{t}\left[U_{1}\left(x_{t}, \frac{M_{t}}{p_{t} e_{t}}\right)\left(\frac{D_{t}^{i}}{p_{t}}\right)\right]
$$

subject to (3), where $\beta^{t} a_{t} U_{1}\left(x_{t}, \frac{M_{t}}{p_{t} e_{t}}\right)$ measures the marginal utility value to the household of an additional unit of profits received at $t$ and real dividends are

$$
\frac{D_{t}^{i}}{p_{t}}=\left(\frac{p_{t}^{i}}{p_{t}}\right)^{1-\theta} y_{t}-\left(\frac{p_{t}^{i}}{p_{t}}\right)^{-\theta}\left(\frac{w_{t} y_{t}}{z_{t}}\right)-\frac{\phi}{2}\left(\frac{p_{t}^{i}}{\pi^{s} p_{t-1}^{i}}-1\right)^{2} y_{t}
$$

The monetary authority is characterized by a set of rules where the policy instrument, which could be either the nominal rate or the growth rate of money, responds to past values of the interest rate and to current values of output, inflation:

$$
0=R_{t}^{-1} R_{t-1}^{\rho_{r}} y_{t}^{\left(1-\rho_{r}\right) \rho_{y}} \pi_{t}^{\left(1-\rho_{r}\right) \rho_{\pi}} \Delta M_{t}^{\left(1-\rho_{r}\right) \rho_{m}} \epsilon_{t}
$$

and $\epsilon_{t}$ is a monetary policy shock.

There are four disturbances in the model $v_{t}=\left(a_{t}, e_{t}, z_{t}, \epsilon_{t}\right)$ and they are characterized by $\log v_{t}=\bar{v}+N \log v_{t-1}+u_{t}$, where $N$ is a diagonal matrix with entries $\left(\rho_{a}, \rho_{e}, \rho_{z}, 0\right)$, respectively. The covariance matrix $\Sigma$ of the structural shocks $u_{t}$ is diagonal, with entries $\sigma_{a}^{2}, \sigma_{e}^{2}, \sigma_{z}^{2}, \sigma_{\epsilon}^{2}$. In a symmetric equilibrium all the firms make identical choices so $y_{t}^{i}=y_{t}, n_{t}^{i}=n_{t}, p_{t}^{i}=p_{t}$, and $D_{t}^{i}=D_{t}$.

Log-linearizing the equilibrium conditions around the steady states leads to:

$$
\begin{aligned}
\hat{y}_{t} & =\frac{1}{1+h} E_{t} \hat{y}_{t+1}+\frac{h}{1+h} \hat{y}_{t-1}-\frac{\omega_{1}}{1+h}\left[\hat{R}_{t}-E_{t} \hat{\pi}_{t+1}-\left(\hat{a}_{t}-E_{t} \hat{a}_{t+1}\right)\right] \\
& +\frac{\omega_{2}}{1+h}\left[\left(\hat{m}_{t}-\hat{e}_{t}\right)-\left(E_{t} \hat{m}_{t+1}-E_{t} \hat{e}_{t+1}\right)\right] \\
\hat{m}_{t} & =\gamma_{1}\left(\hat{y}_{t}-h \hat{y}_{t-1}\right)-\gamma_{2} \hat{R}_{t}+\left(1-\left(R^{s}-1\right) \gamma_{2}\right) \hat{e}_{t} \\
\hat{\pi}_{t} & =\beta E_{t} \hat{\pi}_{t+1}+\psi\left(\frac{1}{\omega_{1}}\left(\hat{y}_{t}-h \hat{y}_{t-1}\right)-\frac{\omega_{2}}{\omega_{1}}\left(\hat{m}_{t}-\hat{e}_{t}\right)-\hat{z}_{t}\right) \\
0 & =-\hat{R}_{t}+\rho_{r} \hat{R}_{t-1}+\left(1-\rho_{r}\right) \rho_{y} \hat{y}_{t-p}+\left(1-\rho_{r}\right) \rho_{\pi} \hat{\pi}_{t-p} \\
& +\left(1-\rho_{r}\right) \rho_{m} \Delta\left(\hat{m}_{t-p}+\hat{\pi}_{t-p}\right)+\hat{\epsilon}_{t}
\end{aligned}
$$

where

$$
\begin{aligned}
& \omega_{1}=-\frac{U_{1}\left(x^{s}, \frac{m^{s}}{e^{s}}\right)}{y^{s} U_{11}\left(x^{s}, \frac{m^{s}}{e^{s}}\right)} \\
& \omega_{2}=-\frac{m^{s}}{e^{s}} \frac{U_{12}\left(x^{s}, \frac{m^{s}}{e^{s}}\right)}{y^{s} U_{11}\left(x^{s}, \frac{m^{s}}{e^{s}}\right)}
\end{aligned}
$$




$$
\begin{aligned}
\gamma_{2} & =\frac{R^{s}}{\left(R^{s}-1\right)\left(m^{s} / e^{s}\right)}\left(\frac{U_{2}\left(x^{s}, \frac{m^{s}}{e^{s}}\right)}{\left(R^{s}-1\right) e^{s} U_{12}\left(x^{s}, \frac{m^{s}}{e^{s}}\right)-R^{s} U_{22}\left(x^{s}, \frac{m^{s}}{e^{s}}\right)}\right) \\
\gamma_{1} & =\left(R^{s}-1+R^{s} \omega_{2} \frac{y^{s}}{m^{s}}\right)\left(\frac{\gamma_{2}}{\omega_{1}}\right) \\
\psi & =\frac{\theta-1}{\phi}
\end{aligned}
$$

The superscript $s$ denotes steady state values of the variables, $U_{j}$ is the first derivative of $U$ with respect to argument $j=1,2$ and $U_{i j}$ is the second order derivative of the utility function, $i, j=1,2$.

The log-linearized Euler condition (equation (8)) and the forward looking Phillips curve (equation (10) include, in addition to standard arguments, terms involving real money balances and the money demand shocks. These terms are irrelevant for (cyclical) output and inflation determination if and only if the utility function is separable in consumption and real balances, i.e. $U_{12}=0$ (see equation (13)). Intuitively, real balances matter in the Euler condition because they affect the marginal rate of substitution between consumption and leisure. Thus, they influence not only the aggregate demand but also the aggregate supply, via intertemporal consumption-saving decisions. Since, in equilibrium, the marginal rate of substitution between consumption and leisure is equal to the real wage, real balances matter in the Phillips curve because the real wage is a crucial component of the marginal costs and directly affects the aggregate supply curve. Interestingly, the model implies that whenever real balances enter the Euler equation and Phillips curve, the money demand shock enters too - these cross equation restrictions help with the identification of the parameters.

In this paper, we are interested primarily in the estimates of $\omega_{2}$ and of $\rho_{m}$. $\omega_{2}$ measures the direct role of money in determining the magnitude and the persistence of output and inflation fluctuations; $\rho_{m}$, on the other hand, measures the long run indirect effects that money has on these two variables, through the actions of the monetary authority. When $\omega_{2}$ is zero, utility is separable and real balances have no direct role in propagating monetary business cycles. When both $\omega_{2}$ and $\rho_{m}$ are zero, money could be totally omitted from the analysis without statistical or interpretation losses. Our a-priori expectation is that both $\omega_{2}$ and $\rho_{m}$ are significantly different from zero but vary over time. Our empirical analysis intends to shed light on this conjecture. 


\subsection{The quantitative role of money}

The previous subsection has indicated how money affects the equilibrium level of output and inflation. This qualitative analysis provides useful intuition but is insufficient to justify the use of monetary models where money has a role. In particular, it gives no evidence on how important money is in amplifying real and nominal fluctuations and it is silent about the quantitative size of the effects. Thus, before estimating the model, we want to explore what difference the presence of money may make for output and inflation fluctuations and along which dimensions we should expect giving money a role may help us to understand better the nature of real world fluctuations.

We plot in figure 1 the responses of output and inflation to unitary impulses in the four disturbances in three different situations: when both $\omega_{2}$ and $\rho_{m}$ are different from zero, when $\omega_{2}$ is different from zero but $\rho_{m}$ is zero and when both parameters are zero. We set $\beta=.99, \psi=0.1, \omega_{1}=2.0, \gamma_{2}=0.72, \sigma_{a}=0.31, \sigma_{z}=0.059, \sigma_{e}=0.009, \sigma_{\epsilon}=$ $0.007 h=0.8, \rho_{r}=0.7, \rho_{y}=0.2, \rho_{\pi}=1.52$, and $\rho_{a}=0.40, \rho_{z}=0.70, \rho_{e}=0.80$, which are close to the values calibrated or estimated by Ireland (2004), and let $\omega_{2}$ to be either 2.0 or 0.0 and $\rho_{m}$ be either 1.0 or 0.0 . These values are arbitrary, but $\omega_{2}=2$ implies relatively small complementarities between real balances and consumption in the utility function and $\rho_{m}=1$ implies a 30 basis point change in the nominal rate for a one percent deviation of real money growth from its steady state path. Both values are in the ball-park set of estimates we provide below.

The figure indicates that money is important to propagate certain type of shocks to output. In particular, when the stock of money plays a role, the response of output to technology and to money demand shocks is stronger and longer lived. For inflation, differences are smaller, but even in this case, the responses are larger when money has a role. Notice that the direct and the indirect channels reinforce each other so that, for example, the sum of the individual effects is typically smaller than the combined effect obtained when both $\rho_{m} \neq 0$ and $\omega_{2} \neq 0$.

The unconditional variabilities and the persistence of output and inflation are also affected by the presence of money. For example, in the model with both channels operating, the (average across 100 simulations) variabilities of output and inflation are 0.0014 and 0.0002 , respectively. These variabilities drop to 0.0011 and 0.00017 when only the direct channel is present, and to 0.00009 and 0.00013 , when money plays no role. Similarly, the persistence of output and inflation fluctuations drops from 0.8 and 
0.75 to 0.68 and 0.57 , when both channels are shut down. We do not want to attach too much emphasis to these numbers since the calibration we have used is purely illustrative. Nevertheless, it is clear that, both conditionally and unconditionally, money may make an important quantitative difference in interpreting real world evidence.

\section{The data and the estimation approach}

We assume that the investigator observes data for output, the inflation rate, the nominal rate and real balances. The sample used to estimate the structural parameters goes from 1980:1 to 2007:4. The data is obtained from IMF and OECD data bases. The inflation rate is measured by the growth rate of the GDP deflator; the nominal rate by the call rate; for money we use M2 plus certificate of deposits. GDP and monetary aggregates are scaled by the GDP deflator and by civilian population in the 16-65 group to transform them in real per-capita terms. The raw data (in logs for scaled GDP and M2) is plotted in figure 2.

One could argue that, since M2 includes interest bearing assets, it is not the most appropriate monetary aggregate to use in the model. We have two responses to this point. First, M2 is the standard aggregate that the business cycle literature has used and this facilitates the comparison of interesting correlation statistics. Second, while smaller monetary aggregates are highly unstable, M2 does not show any evidence of instability. A discussion of the results obtained with an alternative the monetary aggregate is in section 4.5

Per-capita real GDP and real money balances display an upward trend. Since the drift appears to be almost deterministic and idiosyncratic across variables, we separately eliminate it from the log of the two series using a linear specification. The inverted pattern that interest rates and inflation display are much more difficult to deal with. Consistent with the literature, we demean both series.

One alternative to the strategy we use to match the data to the model's counterparts is to allow the technology shock $z_{t}$ to be non-stationary and remove the upward trend in per-capita real output and per-capita real balances using a model consistent methodology. We do not follow this approach for two reasons. First, when technology shocks have a unit root, per-capita output and real balances share the same trend, which is not the case here. Second, it is unclear whether all non-cyclical fluctuations can be safely attributed to non-stationary technology shocks. Chang et al. (2006) have 
recently fit a model with non-stationary preference shocks to US data with good results.

The model (8)-(11) contains 19 parameters; 5 structural ones $\eta_{1}=\left(h, \rho_{r}, \rho_{y}, \rho_{\pi}, \rho_{m}\right)$, 3 semi-structural ones $\eta_{2}=\left(\psi, \omega_{2}, \gamma_{2}\right)$ and 7 auxiliary ones, $\eta_{3}=\left(\rho_{a}, \rho_{z}, \rho_{e}, \sigma_{a}, \sigma_{z}, \sigma_{e}\right.$, $\left.\sigma_{\epsilon}\right)$ plus the discount factor $\beta$, the utility parameter $\omega_{1}$, and the steady state values of the nominal interest rate and the output to real balance ratio, $\eta_{4}=\left(\beta, R^{s}, \omega_{1} \frac{y^{s}}{m^{s}}\right)$. Our exercise is geared to obtain likelihood-based estimates of $\eta=\left(\eta_{1}, \eta_{2}, \eta_{3}\right)$, conditional on selected values for $\eta_{4}$. We read $R^{s}$ off the average level of inflation, once we set $\beta=0.99$. The value of $\omega_{1}$ is set to 2 and $\frac{m}{y}$ ratio is fixed to 1.5, following Chari et. al. (2002). Changing this ratio from 1.5 to 2.5 has minor consequences on the estimates we obtain. We do not estimate $\eta_{4}$ jointly with the other parameters because the likelihood has little information for these parameters - since the variables are detrended (demeaned), one should expect this to happen.

The model can be solved using standard methods. Its solution has the format:

$$
\begin{aligned}
x_{1 t+1} & =A_{1}(\eta) x_{1 t}+A_{2}(\eta) u_{t} \\
x_{2 t} & =A_{3}(\eta) x_{1 t}+A_{4}(\eta) u_{t}
\end{aligned}
$$

where $x_{2 t}=\left[\hat{y}_{t}, \hat{\pi}_{t}, \hat{R}_{t}, \hat{m}_{t}\right], x_{1 t}=\left[\hat{y}_{t-1}, \hat{\pi}_{t-1}, \hat{R}_{t-1}, \hat{m}_{t-1}, \Delta \hat{M}_{t-1}, \hat{v}_{1 t}, \hat{v}_{2 t}, \hat{v}_{3 t}, \hat{v}_{4 t}\right]$ and the matrices $A_{i}(\eta), i=1,2$ are complicated nonlinear functions of the parameters $\eta$.

Likelihood-based estimation of the parameters entering (17) and (18) is simple: given some $\eta$, and a sample $\left[t_{1}, \ldots, t_{2}\right]$, we compute the likelihood, denoted by $f\left(y_{\left[t_{1}, \ldots, t_{2}\right]} \mid \eta\right)$, by means of the Kalman filter and the prediction error decomposition and update the original $\eta$ values using gradient methods. The Kalman filter is easy to use since the solution comes in the form a linear state space system where (17) is a transition equation and (18) an observation equation.

The likelihood function of the model is difficult to maximize since it displays multiple peaks and large flat areas. It is well known that Bayesian methods have an edge over classical methods when the likelihood function is poorly behaved. However, the choice of priors is problematic here since it may make the posterior of the parameters look very different from the likelihood. In this situation inference may crucially depend on the shape, the location and the spread of the multivariate prior density. Our choice of letting the data freely speak is complemented with an ex-post criteria, which eliminates economically implausible estimates or estimates violating stability or non-negativity constraints. 


\section{The results}

\subsection{Full sample evidence}

We start by analyzing the estimates we obtain for the full sample, which we report in the first column of table 1 . In parenthesis are standard errors, computed from the Hessian of the function at the maximum.

Estimation appears to be sufficiently successful and all the economic parameters are significant. Habit in consumption turns out to be strong, as is the inertia in the nominal rate. The response of the nominal interest rate to output is weak and the semielasticity of money demand similar to the one estimated in the US for this period. The money demand shock is highly persistent; the preference and the technology shocks die out sufficiently fast. We plot the (reduced form) residuals of the output and inflation equations in Figure 3, as an additional check on the success of the estimation exercise. The model captures the decline in output and inflation occurred in the 1990s, but falls short of fully explaining the pattern. In fact, the residuals of both variables display a small declining trend from 1990 up to 1995. Thus, the model needs to be augmented with additional features to be able to completely account for the experience.

Turning to the estimates of the parameters of interest, the direct effect of money $\omega_{2}$ is 1.74 and this value is statistically significant and the (long run) indirect effect of money $\rho_{m}$ is 1.50 and it is again statistically significant. We formally test for the joint significance of the direct and indirect effects of money using an asymptotic likelihood ratio test and a small sample version of the same test, where a correction for the number of estimated parameters is used. The null hypothesis in both cases is that money does not matter, i.e. $\omega_{2}=\rho_{m}=0$. All other parameters are left unrestricted under the null and the alternative. The difference in the log-likelihood of the two specifications is large (1451 vs. 1436) and the null hypothesis is soundly rejected with both tests.

It is comforting to find that the effects of money are statistically significant and that the presence of money improves the fit of the model. Still, what matters most is whether money plays an important economic role, where by this we mean that the absence of money would induce researchers to draw wrong conclusions about the drivers of output and inflation fluctuations. To study this question, figure 4 plots the responses of output and inflation to the four disturbances, when the model is estimated with and without money. In a model where money matters, the responses of both output and inflation to technology and money demand shocks are considerably amplified and the magnitude 
and the persistence of the responses to preference and, to some extent, monetary policy shocks are significantly toned down. Hence, models where money dichotomizes with the rest of the variables provide a distorted view of the sources of fluctuations in the economy. For example, technology shocks explain the largest portion of the forecast error variance of inflation at the 4-5 year horizon in the benchmark model (48 percent), but such a proportion dramatically falls when $\omega_{2}$ and $\rho_{m}$ are zero (26 percent) and preference shocks become the dominant source of variations.

In sum, the model appears to do a reasonably good job in characterizing the Japanese economy over the last 30 years. The structural evidence we have collected suggests that money statistically matters in shaping monetary business cycles in Japan. Excluding money from the model affects the statistical fit and may change the economic interpretation of the evidence. Since the persistence and the variability of the responses of output and inflation to exogenous disturbances depend on the postulated role of money, it is possible to attribute certain patterns to the wrong sources, when using a standard specification with no feedback from money to output, inflation or the nominal rate.

\subsection{Subsample evidence}

The analysis in the previous subsection was conducted assuming that no structural changes took place. It is not hard to question such an assumption: there is plenty of evidence that structural changes did occur in Japan over this period. For example, the length of the workweek was reduced at the beginning of the 1990s; the legal status of the Bank of Japan changed at the end of 1997; and the (steady state) level of saving dropped considerably in the 1990s from the level prevailing in the 1980s. To see whether our conclusions concerning the role of money are due to inappropriate handling of these time varying patterns, we repeat structural estimation over several subsamples. The main problem we face in conducting subsample estimation is that a break date is hard to select. The multiplicity of events we mention indicates that it may be difficult to decide a-priori when the new regime started and specification errors may matter.

Rather than arbitrarily choosing a break date, we proceed as in the literature that tests for (unknown) structural breaks and conduct estimation over many subsamples. That is, we start estimating the model over the subsamples 1980-1990 and 1991-2007, then repeated estimation 9 times, augmenting the end date of the first subsample by a year, and concluded estimating the model over the subsamples 1980-1998 and 
1999-2007. We then compute the log-likelihood for each break date (summing the loglikelihoods for each of the two subsamples) and select as break date the point giving the highest log-likelihood. It turns out that the two most likely break dates are 1991 and 1997 and that the difference in the log-likelihood is small (1539 vs. 1537). Since both dates seem to be important, we present structural estimates breaking the full sample at 1991 and 1997 (see table 1, columns 2 to 5). Incidentally, the log likelihood obtained breaking the sample in two is always larger than the log likelihood for the full sample. Therefore, there is a strong statistical evidence that the structure of the Japanese economy changed significantly over the 1990s.

Estimates of the structural parameters tend to change over subsamples. For example, the output coefficient in the Taylor rule is much larger in the 1980-1991 sample or in 1998-2007 sample than in the full sample. Similarly, the semi-elasticity of money demand to interest rates is much larger in the 1980-1991 and 1998-2007 samples than in the full sample. The coefficient on inflation in the Taylor rule (which is here the sum of $\rho_{p}$ and $\rho_{m}$ ) fluctuates somewhat depending on the sample and in the 1992-2007 is very close to the lower bound of admissible values we have chosen to insure that no indeterminate solutions occur. Interestingly, while the persistence of technology shocks is large in both the 1980-1991 and the 1980-1997 samples, it becomes negligible in the 1992-2007 and 1998-2007 subsamples. Finally, there are important changes in the relative variability of the shocks over time: technology shocks dominate in the 1980s, preference shocks have the largest volatility in the 1990s and, in the 2000s, the variability of technology shock is once again the largest. Because of the small size of subsamples, standard errors are typically large and most of the estimates of the economic parameters turn out to be insignificant.

Turning to the parameters of interest, we find that estimates of $\omega_{2}$ and $\rho_{m}$ vary over subsamples. In particular, the importance of the indirect effect of money $\rho_{m}$ steadily declines - the point estimate we obtain in the second subsamples is about half of the point estimate obtained in the first subsamples, no matter what break date we consider. This pattern appears to be consistent with the conventional wisdom that money was an important determinant of monetary policy decisions in the 1980s and that its importance declined considerably since then. Also interestingly, from our point of view is the fact that the direct effect of money is small in the 1980s, becomes more important in the 1990s and disappears in the 2000s. Thus, having money in the model seems to be important when explaining the mechanisms that led to the "Lost decade" 
but probably not so crucial to account for the 2000s. This conclusion is consistent with the reduced form evidence of Miyao (2005), who find that the link between monetary aggregates, output and prices disappears in the late-1990s.

We plot the responses of output and inflation to the four shocks in the full sample and in the subsamples 1980-1991, 1992-2007 and 1998-2007 in figure 5. At naked eye, one can notice significant changes in the transmission of disturbances over time. For example, output responses to monetary policy and technology shocks are stronger and less persistent in the last sample - here the half life of the responses to both shocks is about half of the size as with the other two subsamples - and output responses to money demand shocks are also much stronger in the last sample. One should be careful in interpreting this evidence as indicating that monetary policy shocks have become more powerful in the last part of the sample, because the variance of the shocks may have changed. In fact, as we see from table 1, the variance of the shocks driving the monetary policy rule fall considerably in the last 10 years. Hence, the fivefold increase in the impact effect of monetary shocks on output is more than compensated by the 9 fold decline in the variance of the shocks, making monetary policy, if anything, less effective in the 1998-2007 period (see e.g. Miyao (2000) and Inoue and Okimoto (2008) for VAR evidence on this issue).

Variations over time in the responses of inflation are generally much smaller. However, one can notice a more persistent response to preference shocks in the last 10 years and a progressively weaker but more persistent response to monetary policy shocks, as we move from the 1980s to the latter part of the sample. Hence, while the impact effect of monetary policy on inflation has consistently declined, it appears that (bad) shocks can affect it for a longer period.

Two other interesting features of output responses should be mentioned. First, the responses of output to the disturbances in the 1980-1991 and 1992-2007 samples are similar. Therefore, even though, statistically, the 1991 break is the strongest, there are limited economic differences in the two selected subsamples - an indication that it is the distribution of the shocks which has changed at this date. Second, full sample responses tend to be stronger and more persistent than subsamples responses. Thus aggregation biases may be important, making the practice of interpreting the 1990s with full sample estimates not very credible.

Finally, one can easily see from figure 5 that technology shocks are the most important driver of the forecast error variance of inflation in every subsample at medium-long 
term horizons. Preference shocks, and to a much smaller extent technology shocks, are instead the main determinants of the forecast error variance of output in all subsamples. Hence, the output stagnation of the 1990s is not necessarily connected with the long deflation and to account for the two phenomena at least two sources of disturbances are needed. We will come back on this issue in the next section.

\subsection{How does the model account for the lost decade?}

We have seen that the simple model we employ is, to a large extent, capable of replicating the decline of output and the deflation experienced by the Japanese economy during the 1990s. The question we want to investigate here is how does the model capture these changes. In particular, we are interested in knowing which set of parameters is altered and whether most of the variations occur in the economic parameters or in the auxiliary parameters describing the time series process for the disturbances. Apart from shedding light on the mechanics of the "Lost decade", as seen through the lenses of our model, such an investigation may provide further evidence on whether the changes experienced in industrialized economies over the last 30 years are due to variations of the structure or to changes in the type and intensity of the disturbances.

It turns out that, on the private sector side of the economy, $\omega_{2}$ and $\gamma_{2}$ are the parameters which vary most across subsamples. The monetary policy rule seems also to have changed significantly: interest rate inertia is stronger and both the inflation and the output parameters are affected. However, the largest changes appear to occur in the parameters describing the stochastic process for the exogenous disturbances. For example, the persistence and the variability of the preference shock vary considerably over time and the variability of the technology shocks also changes across subsamples. This conclusion is roughly independent of which of the two break dates one considers. Thus, the model tells us that both the structure of the economy and the nature of the shocks have changed, a finding which is consistent with the most recent structural literature on the Great Moderation in the US (see e.g. Canova, 2009). To examine which variation matters most we conduct two counterfactuals where we fix one set of parameters (either the economic or the auxiliary ones) at the level prevailing in the first subsample and let the other set be freely estimated in the second subsample. We then compare the likelihood obtained when all the parameters are left unrestricted with those obtained in the two restricted specifications. Restricting either set of parameters to be unchanged significantly affects the log likelihood - the drop is by more than 60 
points, regardless of the chosen break date. However, when we restrict the distribution of the shocks to be time invariant, the drop is larger by about 30 points. Hence, changes in the distribution of the shocks are more important to account for the Lost Decade.

\subsection{Discussion}

A few important lessons can be drawn from our analysis. First, the stock of money is statistically important in characterizing cyclical fluctuations in output and inflation in Japan and both the direct and the indirect effects of money are crucial to understand how cyclical fluctuations are propagated. As far as we know, the typical policy rules that are nowadays estimated in the literature do not include the growth rate of nominal balance among the regressors. Since reacting to nominal balances is also a way to indirectly react to inflation, it is likely that, in typical rules, the inflation coefficient is overestimated, the richness of second round dynamics due to money muffled, and policy conclusions distorted.

Second, the role of money has changed over time and our estimates indicate that money may have mattered most in the 1990s. While this outcome may indicate misspecification - money may be proxying in the 1990s for mechanisms which are missing from the model - we have found no evidence that money stands in for standard omitted suspects (asset prices, oil, etc.). For example, repeating estimation allowing for oil in the production function does not change any of the conclusions. Interestingly, while structural estimates change across samples, the transmission of shocks to inflation hardly changes over time. On the other hand, the transmission mechanism of disturbances to output shows some variations, but only since 1998. Hence, the changes that the Japanese economy experienced started producing interesting economic effects only in the last 10 years and these changes make the real side of the economy temporarily more reactive to shocks.

Third, the "Lost decade" is a complex experience and our model accounts for it with changes in the parameters regulating the private sector behavior, changes in the parameters of the monetary policy rule, changes in the distribution from which disturbances are drawn and residual unexplained variations. While all contribute, changes in shock distribution are the most important ones. This conclusion is in part due to the simplicity of the model which does not allow, for example, for an intercept in the Taylor rule (as in Braun and Waki, 2006) or to distinguish between changes in efficiency vs. changes in the technological progress (such as Nemoto and Goto, 2005). Had a 
more elaborate structure been considered, the relative importance of various sources of variations could have been altered.

\subsection{Robustness}

In this subsection we discuss the results of two sensitivity exercises: one designed to investigate how misleading is our specification for monetary policy when the interest rate reached the zero lower bound; and another to study the effects of changing the monetary aggregates on estimates of the parameters of interest.

When the interest rate reaches the zero bound, it may be difficult to claim that a standard Taylor rule describes well the monetary policy process. Therefore, one may consider adding a zero lower bound constraint to the model, as in e.g. Braun and Waki (2006). However, maximum likelihood estimation of a model where the zero bound may be occasionally binding is unfeasible because the decision rules of the model imply that the likelihood function can no longer be computed with the Kalman filter.

However, our specification is sufficiently flexible that can endogenously adapt to situations where the zero lower bound is hit without any need to take the bound explicitly into account. To see this consider the point estimates obtained in the subsample 19982007. From table 1 one can see that the estimated rule can be approximately written as $R_{t}=0.5 y_{t}+0.4 \pi_{t}+\Delta m_{t}+u_{t}$ where $u_{t}$ is a process with long memory. Since $R_{t}$ is roughly zero over the sample, the rule can be alternatively written as an implicit money growth rule: $\Delta m_{t}=-0.5 y_{t}-0.4 \pi_{t}-u_{t}$. Hence, with the estimated parameters, the rule tells us that money growth increases when the gap is negative and when inflation goes into negative territory - something very much akin to quantitative easing. Interestingly, and confirming the conventional wisdom, the estimated coefficients imply that the reaction of money growth to economic growth is weak - an indication that over the last 10 years a policy of quantitative easing fell shy of the need of the economy.

To restate the point in a somewhat different way, when the zero bound on interest rate is reached, our policy rule can be transformed into a money growth rule without any need to change the specification or to directly specify a lower bound on the interest rate. The data endogenously selects the most appropriate normalization for each sample.

As we argued, our main reason for using an aggregate like M2 plus certificate of deposits is that this series, once it is normalized by the price level and by population, is much more stable and with much more reasonable properties than other smaller monetary aggregates. For example, while per-capita real M2 balances and real M1 
balances have a similar trends (long run correlation 0.93), their cyclical components are substantially different. In fact, their contemporaneous correlation is negative (0.45). Moreover, the contemporaneous correlation of the cyclical component of percapita real M2 balances and output is about 0.7 , while the contemporaneous correlation of the cyclical component of per-capita real M1 balances and output is -0.77 . The counterintuitive sign for this latter correlation is roughly maintained if we split the sample in two, even though the magnitude of the correlation changes considerably (from -0.85 for the sample up to 1991 to -0.31 afterward). However, since it is not clear which monetary aggregate belongs to the utility function of our representative agent, we repeated estimation over the subsamples 1980-1997 and 1997-2008 using real per-capita M1 balances. The results are in the last two columns of table 1 and do not appear to be very reasonable. For example, both $\omega_{2}$ and $\rho_{m}$ are negative in both samples, although only the former is significant; the coefficient on inflation in the Taylor rule is higher in the second subsample and estimates $\psi$, which are related to price stickiness of the economy, increase dramatically in the second subsample. In addition, the fit of the model appear to be unsatisfactory - the residuals of the output and inflation equations are serially correlated and display a slight downward trend - reinforcing the conclusion that M2 should be used in our structural estimation exercises.

\section{What drives the path of output and inflation in the 1990s?}

The depressing performance of Japan in recent years has provided the stimulus for extensive academic research (see, among others, the symposium issues of the Japanese Economic Review, 2006, and the Journal of Japanese and International Economics, 2005). The literature appears to dived into two main camps; roughly, one camp emphasizes failures in the supply side; the other attributes the slump to weak demand conditions. Since the performance of our structural model is, by statistical standards, reasonable, we can contribute to the debate by analyzing the contribution of each the four disturbances to the path of output and inflation that materialized over the 1990s. While a decomposition in demand vs. supply driven effects is not possible, since the general equilibrium nature of the model implies that disturbances typically have effects on both the aggregate supply and the aggregate demand curve of the economy, such an analysis is nevertheless useful to highlight sources of output stagnation and of 
the deflation and to provide new insights about the relative validity of the suggested explanations. In figures 6 and 7 we present the results of an historical decomposition exercise where we plot the path of output and inflation which is unpredictable based on the information available at 1991:4 or 1997:4 (dashed line) and the counterfactual path of the same two variables that would have materialized if only one disturbance were present in the economy. In other words, we plot what output and inflation would have been, once the path which is predictable based on the information available at 1991:4 and 1997:4 has been removed, had there be only one type of shock in the period. For readability, we present the paths due to the preference shocks (a-shock), the monetary shock (r-shock) and the technology shock (z-shock) since the contribution of money demand shocks is everywhere negligible.

Figure 6 indicates that the model interprets the downward trend in the unexpected output in the 1990s as primarily due to preference shocks. Monetary policy also contributes to this downward trend, but only since 1997. Interestingly, the counterfactual output path induced by the technological disturbances does not line up well with the idea that technology shocks are responsible for the "Lost decade": output would have been counterintuitively increasing up to the mid-1990s and would have been falling while remaining on the positive territory after that date. Note also that preference shocks are responsible not only for the downward trend but also for most of the short term movements in the unexpected output. Monetary policy and technology shocks instead have no role in explaining unpredictable short term output movements. Technology shocks account for both the downward trend in the path and for the short terms ups and downs in the inflation series (in agreement with the findings of Shimpo (2005)). Preference and monetary disturbances, instead, have little to do both with the path and the fluctuations of the unpredictable component of inflation throughout the 1990s.

Figure 6 sheds some important light on the role of monetary policy in the experience. The Bank of Japan has been often criticized for being behind the cycle and for not having done enough to stimulate the economy. Our structural analysis indicates that monetary policy was expansionary up to the middle of the 1990: both the counterfactual path of unexpected output and inflation would have been positive and substantially so until 1997. Monetary policy turned contractionary since 1998 and both the counterfactual path of output and inflation display a U-shaped pattern since then, with the through located in 2003 - here, the unexpected path of both variables becomes negative. Since 2003, monetary policy has been marginally less restrictive and, by the 
end of the sample, the counterfactual unexpected path of output and inflation due to monetary disturbances is roughly zero. Interestingly, this latest period of less restrictive monetary policy coincides with the period of quantitative easing adopted by the Bank of Japan (see e.g. Ito and Mishkin, 2004).

The results we obtain looking at the last ten years of data, but using estimates obtained up to 1997 (see figure 7) are consistent with what we have found in figure 6. The medium term trends in the unexpected output path since 1998 are due to monetary shocks and technology shocks play no role in driving short or medium run fluctuations. The short term movements in the unpredictable component of inflation are instead mainly driven by technology shocks while preference shocks contribute to keep the level of unexpected inflation gravitates in the negative territory.

Three important conclusions can be derived from this analysis. First, the (real) recession and the (nominal) deflation of the 1990s are driven by different causes. Shocks that affect the (average) intertemporal marginal rate of substitution in the economy, are crucial to explain the path of unexpected output in the 1990s. While the model is too stylized to say more about what these shocks are, one can associate these disturbances with shocks that have changed the average saving rate of the economy and/or have affected the labor supply of the agents. Unexpected inflation movements, instead, appear to be driven by disturbances affecting the aggregate marginal costs of production. Again, since the model is stark, not much can be said about the nature of these disturbances. However, one can guess that they could proxy for fiscal policy shocks, labor supply shocks as well as shocks to the acquisition of goods used in production.

Second, technology shocks have little to do with the long stagnation of the 1990s. It is well known, at least since Evans (1992), that Solow residuals are poor proxies for technological variations and the results we obtain are consistent with this view: Solow residuals, for example, may proxy for the effects captured here by preference disturbances. It is hard to formally compare the Solow residuals produced, e.g., by Hayashi and Prescott (2002) with our technology shocks for a number of reasons. First, their accounting exercises use annual data while our estimates are quarterly. Second, our model has no capital accumulation, while their exercises use data on investment to construct a measure of Solow residuals. Third, they use data only up to the 1990s while our estimates incorporate information up to 2007. Finally, the estimates of $\phi$ we obtain imply, for any reasonable value of the elasticity of substitution between varieties $\theta$, that price stickiness is an important feature of the data. Therefore, the fact that 
Solow residuals are obtained in a frictionless economy while our estimates of technology shocks result from an economy where pricing frictions are important makes a formal comparison of the two measures difficult.

Third, monetary policy is responsible for the poor macroeconomic outcomes of the last ten years. Monetary policy has been very restrictive from 1998 up to 2003 and this has contributed to keep both the recession and the deflation going for a number of additional years. The situation appears to have marginally changed since 2003 and the upward trend that the unexpected output path displays in the last few years is almost entirely due to monetary policy shocks. Thus, there is an important lesson to be learned: a policy of sustained quantitatively easing may be the way to bring the economy out of a recession at times when the nominal interest rate hits the zero bound.

\section{Conclusions}

This paper examines whether money had a role in shaping monetary business cycles in Japan. The theoretical framework of analysis is a small scale structural New Keynesian model where the stock of money potentially enters the utility function and the monetary policy rule. We estimate the parameters using a maximum likelihood technique. We find likelihood based techniques preferable to GMM and similar methods because they take into account the whole system of equations in the estimation and provide a natural framework to test restrictions on the specification we employ. We refrain from using a-priori restrictions on the parameter space to make the information content of the data as transparent as possible.

We study four questions. First, does money have a role in explaining fluctuations in output and inflation in Japan since 1980? Second, is there a change in the importance of money over interesting subsamples? Third, does the model accounts for the Lost Decade via changes in the economic parameters or via changes in the distribution of the shocks? Fourth, what disturbances could be jointly responsible for the output stagnation and the deflation observed in the 1990s?

We find that money is statistically and economically important in characterizing cyclical fluctuations in output and inflation in Japan. Money matters both directly, through its effects on the Euler equation and the Phillips curve, and indirectly, through the Central Bank determination of the nominal interest rate. Money plays a role in the model because it alters both the marginal rate of substitution between consumption and 
leisure and the intertemporal rate of substitution of consumption at different points in time and because, when reacting to nominal balances, the monetary authority indirectly reacts to inflation. These three channels help to amplify the magnitude of cyclical fluctuations that the model can account for and stretch their persistence over time. Our evidence suggests that neglecting money may create important statistical biases and relevant inferential mistakes in interpreting sources and transmission of cyclical fluctuations, in general, and the 1990s in Japan, in particular.

We detect changes in the role of money over time and our estimates indicate that money may have mattered most in the 1990s. While this outcome may be taken as a sign of misspecification, we have found no evidence that money stands in for standard omitted suspects such as asset prices, oil, etc.. Thus, it appears that the quantity of money in circulation may have contributed to transform the downturn of the beginning of the 1990s into a severe output stagnation and a moderate inflation into a deflation.

Our model accounts for Japan's Lost Decade through changes in the economic parameters regulating the private sector behavior and the monetary policy rule and in the auxiliary parameters regulating the persistence and the volatility of the exogenous disturbances. However, changes in the distribution of the shocks are a much more important mechanism to account for the experience than changes in economic parameters. This is consistent with what the literature on the Great Moderation in the US has found using similar small scale structural models (see e.g. Canova, 2009).

Finally, the prolonged fall in output and the persistent deflation experienced in Japan in the 1990s appear to be due to different causes. Shocks that affect the intertemporal marginal rate of substitution in the economy are crucial to explain the path of output. Shocks that alter the marginal cost of production, on the other hand, primarily drive inflation movements. Interestingly, technology shocks have little to do with the long output stagnation of the 1990s. Since it is well known that Solow residuals are poor proxies for technological variations, the idea that TFP changes are responsible for Japan's lost decade should be probably reconsidered. Finally, monetary policy is to blamed for the poor macroeconomic outcomes, at least since 1998. Monetary policy has been very restrictive up to 2003, and this has contributed to keep both the recession and the deflation going for a number of additional years. The situation appears to have changed since 2003, but only marginally.

While we consider our investigation successful, and think that the lessons it provides are useful and appealing to students of the Lost Decade, three caveats needs to be 
mentioned. First, the model used to interpret the data is highly stylized and it is possible that money proxies for other important omitted influences. We have checked if some standard suspects could matter and found no evidence in favor of it. However, general conclusions cannot be drawn unless a larger and less stylized model is used to analyze the experience. Future work in the area should consider this a priority. Second, while the role of money seemed to have changed over time, estimates over subsamples are not statistically significant because the size of samples is limited and the amount of noise present in the data large. Ideally, one would like to go back to the beginning of the 1970s to properly estimate the model over subsamples. Data limitations, however, prevent us to do more in that direction. Third, while we have stressed that certain conclusions crucially depend on giving money a proper role in the economy, others are not influenced by this feature. Disentangling which aspects of the model economy could be affected more by the presence of money, should also be a priority for those interested in measuring the contribution of money to medium term business cycle fluctuations.

\section{References}

[1] Ariga, K., Hayashi, F., and Horioka, C., 2006. Symposium on Macroeconomic Analyses of Japan's Lost Decade: an introduction, The Japanese Economic Review, $57,157-160$

[2] Braun, A. and Waki, Y., 2006. Monetary Policy during Japan's lost decade, The Japanese Economic Review, 56, 324-344.

[3] Caballero, R., Hoshi, T., and Kashyap, A., 2008. Zombie Lending and Depressed Restructuring in Japan, forthcoming, American Economic Review.

[4] Canova, F., 2009. What explains the Great Moderation in the US? A Structural Analysis, Journal of the European Economic Association, 7(4),1-25.

[5] Chang, Y., Doh, T.,. and Schorfheide, F. (2006), Non-stationary Hours in a DSGE model, Journal of Money, Credit and Banking, 39(6), 1357-1373.

[6] Chari, V.V., Kehoe, P. and McGrattan , E., 2002. Can sticky prices generate volatile and persistent real exchange rates, Review of Economic Studies, 69, 533563.

[7] Comin, D., 2008. An exploration of the Japanese Slowdown during the 1990s, NBER working paper 14509. 
[8] Eggertsson, G. and Woodford, M., 2003. The zero bound on Interest Rates and Optimal Monetary Policy, Brookings Papers on Economic Activity, 1, 1-105.

[9] Estaban Pretel, J., Nakayama, R., and Tanaka, R., 2008. TFP Growth Slowdown and the Labor Market in the Medium Run, University of Tokio, manuscript.

[10] Evans, C., 1992. Productivity Shocks and the Real Business Cycle, Journal of Monetary Economics, 29, 191-208.

[11] Gali, J., 2008. Monetary Policy, Inflation, and the Business Cycle: An Introduction to the New Keynesian Framework, Princeton University Press, Princeton, N.J.

[12] Hayashi, F. and Prescott, E., 2002. Japan in the 90s: A Lost decade, Review of Economic Dynamics, 5, 206-235.

[13] Hoshi, T. and Jorgenson, D., 2005. Enhancing Productivity: editor's introduction, The Journal of the Japanese and International Economies, 19, 457-459.

[14] Krugman, P., 1998. It is Baaack: Japan's slump and the return of the liquidity trap, Brookings paper on Economic Activity, 2, 137-205.

[15] Inoue, T., and Okimoto, T., 2008. Were there structural Breaks in the effects of Japanese Monetary Policy? Re-evaluating policy effects of the last decade, Journal of the Japanese and International Economies, 22, 320-342.

[16] Ireland, P., 2004. Money's role in the Monetary Business Cycle, Journal of Money, Credit and Banking, 36, 969-983.

[17] Ito, T. and Mishkin, F., 2004. Two Decades of Japanese Monetary Policy and the Deflation Problem, NBER working paper 10878.

[18] Miyao, R., 2000. Use of Money supply in the conduct of Japan's Monetary Policy: re-examining the time series evidence, Journal of the Japanese and International Economies, 14, 366-384.

[19] Miyao, R., 2005. The Role of Monetary Policy in Japan: A Break in the 1990s, Japanese Economic Review, 56, 165-187.

[20] Nemoto, J. and Goto, M., 2005. Productivity, Efficiency, Scale Economies and Technological Change. A new decomposition analysis of TFP applied to Japanese Prefectures, Journal of the Japanese and International Economies, 19, 617-634.

[21] Shimpo, K., 2005. Interindustry effects of productivity growth in Japan, 1960-2000, The Journal of the Japanese and International Economy, 19, 568-585. 


\begin{tabular}{|c|c|c|c|c|c|c|c|}
\hline & \multicolumn{5}{|c|}{ Using M2 } & \multicolumn{2}{|c|}{ Using M1 } \\
\hline & $1980-2007$ & 1980-1991 & 1980-1997 & $1992-2007$ & 1998-2007 & $1992-2007$ & 1998-2007 \\
\hline$\psi$ & $0.035(0.007)$ & $0.199(0.345)$ & $0.005(0.003)$ & $0.099(0.230)$ & $0.049(0.171)$ & $0.303(0.098)$ & $12.785(0.875)$ \\
\hline$\omega_{2}$ & $1.740(0.499)$ & 0.266 (1.927) & $2.164(0.952)$ & $0.489(3.473)$ & $-0.043(2.268)$ & $-0.372(0.136)$ & $-0.396(0.057)$ \\
\hline$\gamma_{2}$ & $0.696(0.249)$ & $1.512(3.140)$ & $0.567(0.312)$ & $0.508(3.071)$ & $1.948(3.601)$ & $6.006(0.634)$ & 7.859 (1.287) \\
\hline$h$ & $0.990(0.003)$ & $0.884(0.069)$ & $0.989(0.003)$ & $0.979(0.061)$ & $0.878(0.442)$ & $0.986(0.003)$ & $0.639(0.166)$ \\
\hline$\rho_{r}$ & $0.819(0.020)$ & $0.668(0.530)$ & $0.835(0.043)$ & $0.869(0.200)$ & $0.972(0.095)$ & $0.980(0.005)$ & $0.950(0.011)$ \\
\hline$\rho_{y}$ & $0.094(0.075)$ & $0.753(1.974)$ & $0.036(0.036)$ & 0.123 & $0.511(3.116)$ & $0.356(0.356)$ & $-0.192(0.166)$ \\
\hline$\rho_{p}$ & $0.112(0.702)$ & $-0.920(1.849)$ & $0.535(0.860)$ & $0.001(4.702)$ & $0.434(4.569)$ & $1.464(0.509)$ & $2.028(0.570)$ \\
\hline$\rho_{m}$ & $1.508(0.642)$ & $2.181(3.570)$ & $(0.960)$ & $1.006(2.484)$ & $0.962(3.345)$ & $-0.160(0.203)$ & $-0.006(0.133)$ \\
\hline$\rho_{a}$ & $0.252(0.113)$ & $0.750(0.167)$ & $0.179(0.273)$ & $0.323(1.982)$ & $0.648(0.834)$ & $0.345(0.140)$ & $0.882(0.017)$ \\
\hline$\rho_{e}$ & $0.994(0.002)$ & $0.934(0.075)$ & $0.963(0.026)$ & $0.897(0.321)$ & $0.901 \quad(0.365)$ & $0.918(0.021)$ & $0.903(0.021)$ \\
\hline$\rho_{z}$ & $0.640(0.075)$ & $0.907(1.180)$ & $0.975(0.010)$ & 0.048 & $0.020(1.830)$ & $0.159(0.190)$ & $0.759(0.068)$ \\
\hline$\sigma_{r}$ & $0.005(0.113)$ & $0.009(1.082)$ & $0.007(0.102)$ & $0.003(1.805)$ & $0.001(1.636)$ & $0.001(0.169)$ & $0.001(0.252)$ \\
\hline$\sigma_{a}$ & $0.420(0.288)$ & $0.080(1.275)$ & $0.427 \quad(0.596$ & $0.179(3.213)$ & $0.034(2.468)$ & $0.217(0.237)$ & $0.009(0.286)$ \\
\hline$\sigma_{e}$ & $0.008(0.100)$ & $0.015(1.496)$ & $0.009(0.129)$ & $0.005(1.154)$ & $0.006(1.540)$ & $0.025(0.142)$ & $0.025(0.119)$ \\
\hline$\sigma_{z}$ & $0.220(0.324)$ & $0.100(2.029)$ & $0.150(0.119)$ & $0.151(3.230)$ & $0.285(4.156)$ & $0.045(0.461)$ & $0.002(0.152)$ \\
\hline
\end{tabular}

Table 1: Estimated coefficients and standard errors, various samples. 

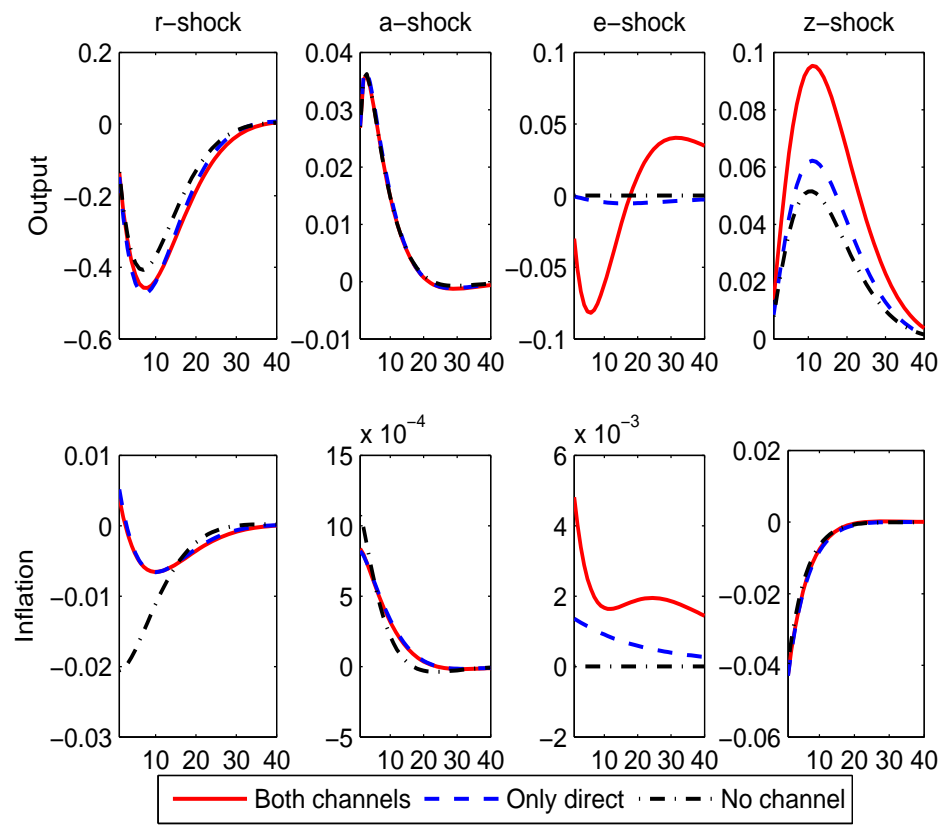

Figure 1: The role of money in the model 

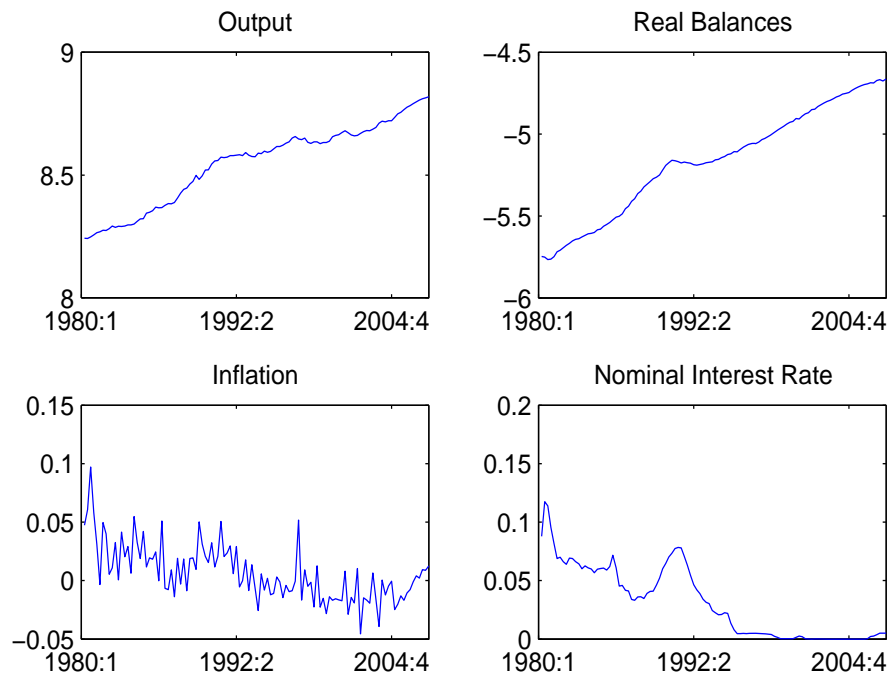

Figure 2: The data 

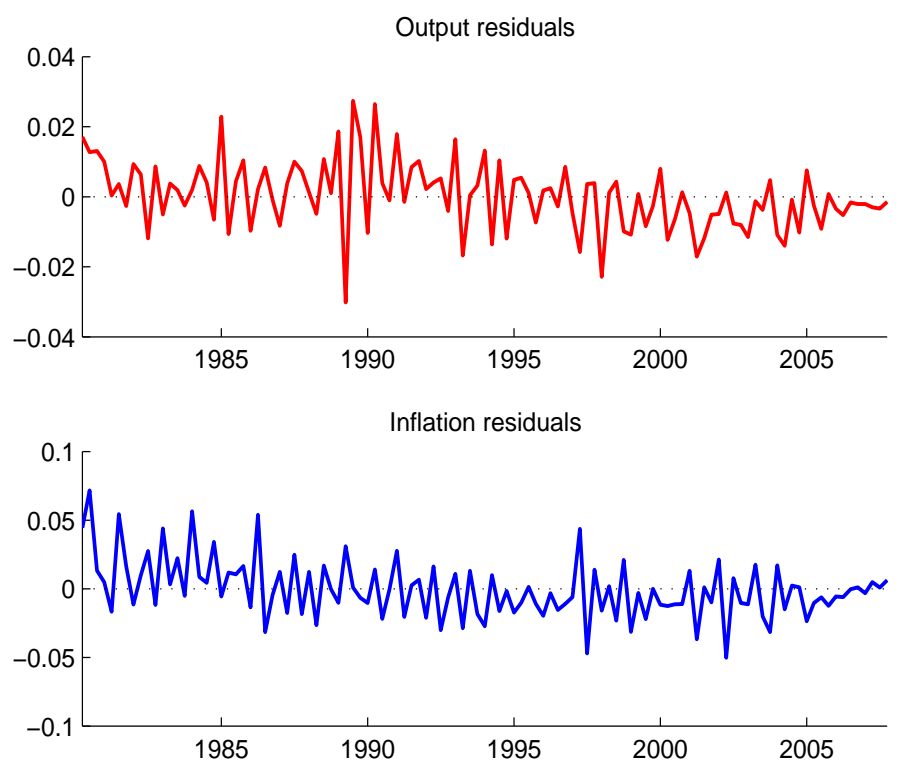

Figure 3: Reduced form residuals 

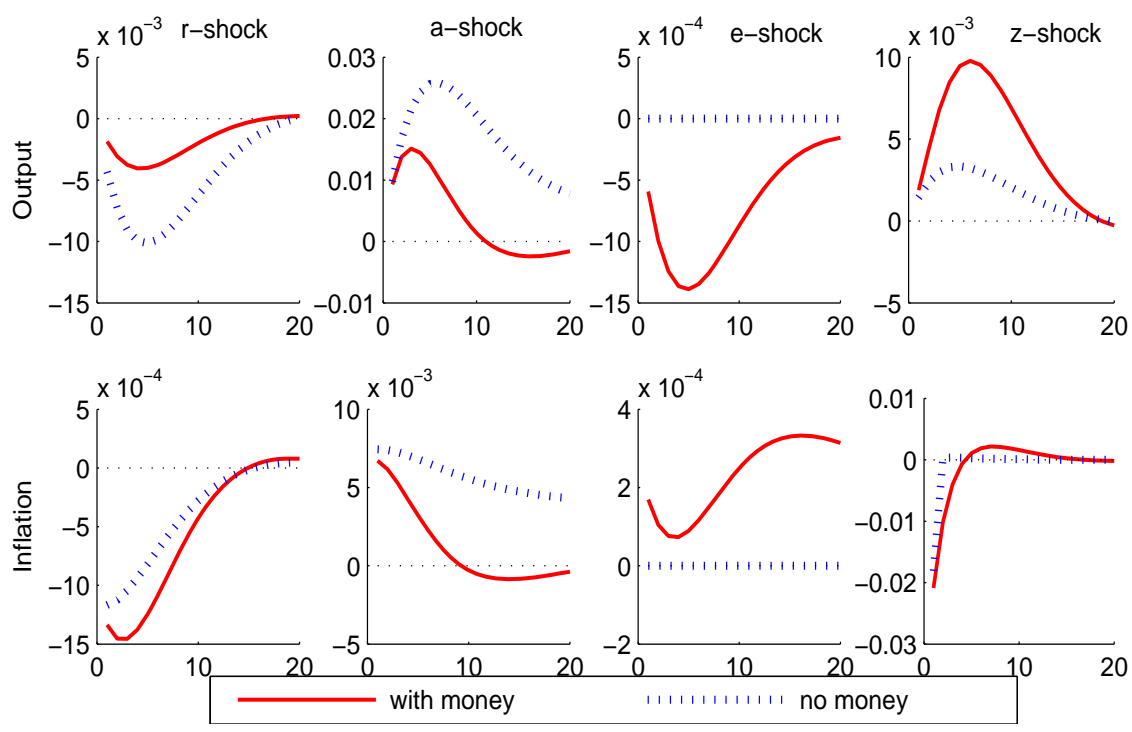

Figure 4: Impulse responses, full sample 

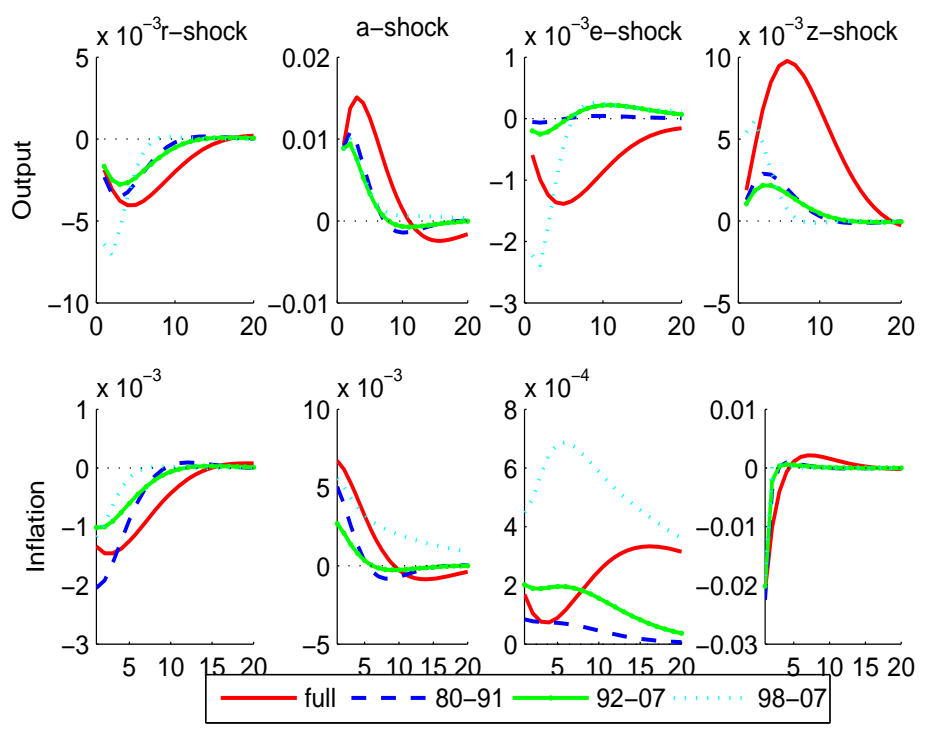

Figure 5: Impulse responses 


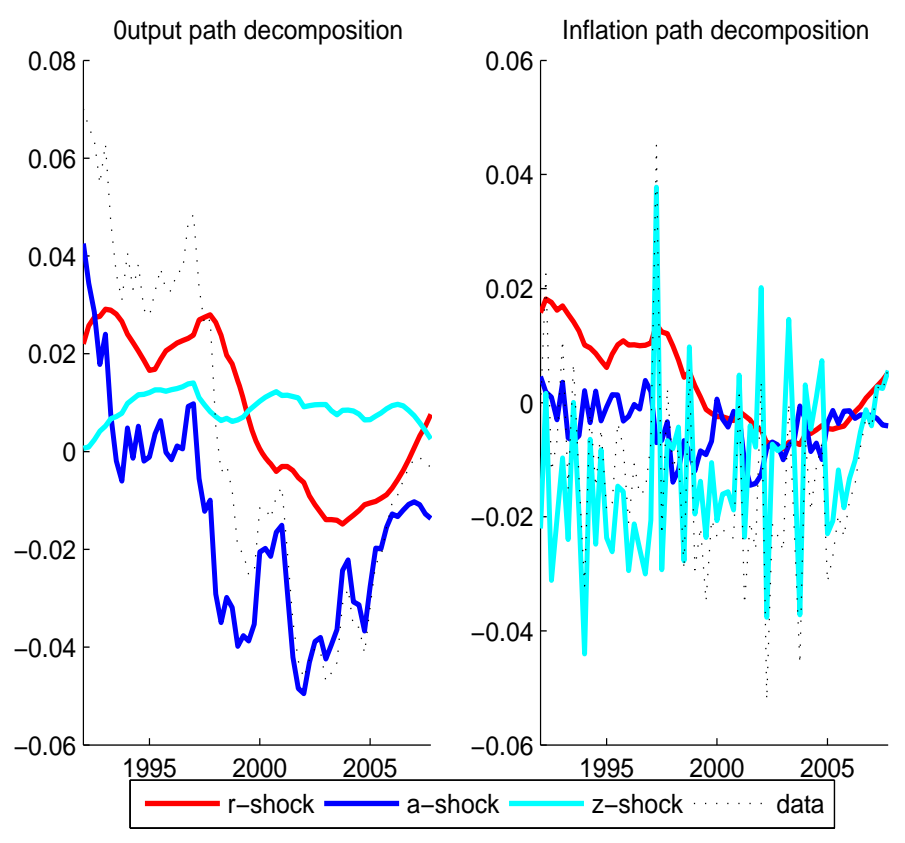

Figure 6: Counterfactual paths, estimates up to 1991:4 


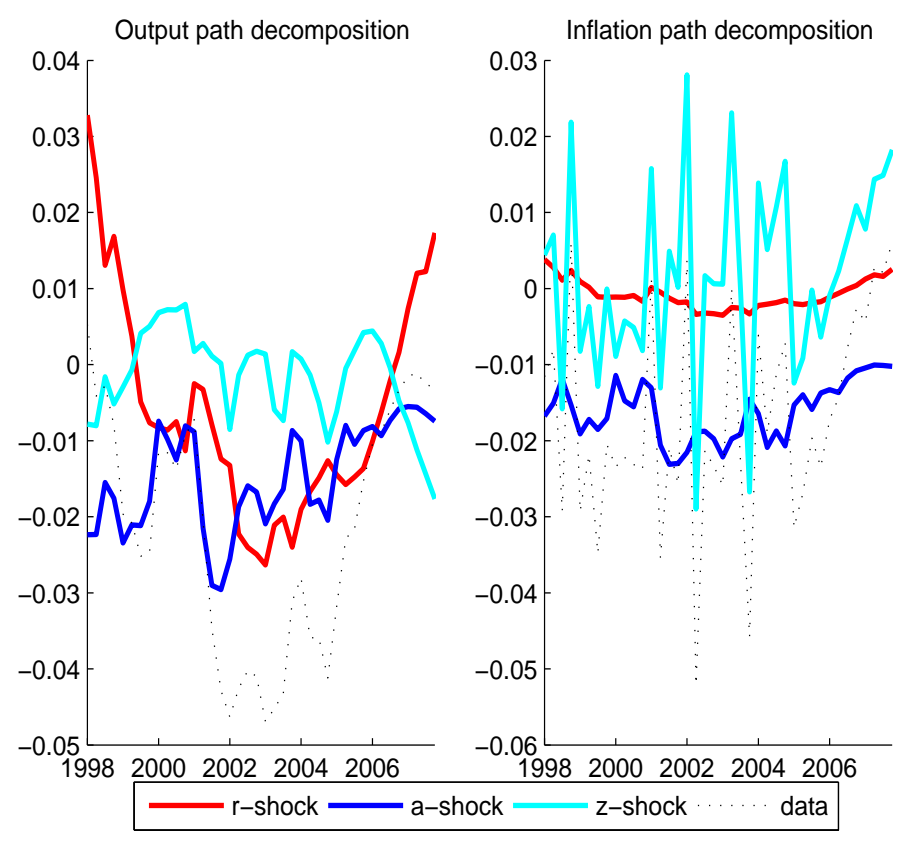

Figure 7: Counterfactual paths, estimates up to 1997:4 